\title{
CORRIGENDUM TO "HARDY TYPE INEQUALITY IN VARIABLE LEBESGUE SPACES"
}

\author{
Humberto Rafeiro and Stefan Samko \\ Universidade do Algarve, Departamento de Matemática \\ Campus de Gambelas, 8005-139 Faro, Portugal; hrafeiro@ualg.pt \\ Universidade do Algarve, Departamento de Matemática \\ Campus de Gambelas, 8005-139 Faro, Portugal; ssamko@ualg.pt
}

\begin{abstract}
In this note, we correct a gap made in the assumptions of the final results of the paper [1]. We give these results with corrected formulations and provide a proof of some auxiliary result which allows us to simultaneously admit regions more general than used in [1].
\end{abstract}

\section{Introduction}

In Theorem 12 of [1] there was lost the assumption that the exterior of the domain should satisfy the cone condition. We correct this gap, and even more, improve the corrected result replacing the cone condition by a weaker assumption on the measure of the intersection of this exterior with balls, called the $A^{c}$-condition.

\section{Auxiliaries}

In Lemma 2 below we give an improvement of Proposition 13 from [1] which allows us to replace the cone condition by the $A^{c}$-condition. It concerns the equivalence of the function

$$
a_{\Omega}(x)=\int_{\mathbf{R}^{n} \backslash \Omega} \frac{d y}{|x-y|^{n+\alpha}}, \quad x \in \Omega
$$

to the function $[\delta(x)]^{-\alpha}$ as $x \rightarrow \partial \Omega$, where $\delta(x)=\operatorname{dist}(x, \partial \Omega)$.

Definition 1. A bounded open set $\Omega \subset \mathbf{R}^{n}$ is said to satisfy the condition $A^{c}$, if there exists a constant $A>0$ not depending on $x_{0} \in \partial \Omega$ and $r>0$ such that $\left|B\left(x_{0}, r\right) \cap\left(\mathbf{R}^{n} \backslash \Omega\right)\right| \geqq A r^{n}$.

Lemma 2. Let $\Omega$ be an arbitrary open bounded set in $\mathbf{R}^{n}$. Then $a_{\Omega}(x) \leqq$ $\frac{c_{1}}{[\delta(x)]^{\alpha}}, c_{1}=\frac{\left|\mathbb{S}^{n-1}\right|}{\alpha}$. If the open set $\Omega$ is of type $A^{c}$, then there exists a constant $c_{2}>0$ such that

$$
\frac{c_{2}}{[\delta(x)]^{\alpha}} \leqq a_{\Omega}(x) \leqq \frac{c_{1}}{[\delta(x)]^{\alpha}}, \quad x \in \Omega,
$$

where $c_{2}=2^{-n-\alpha} A$ and $A$ is the constant from the condition $A^{c}$.

Proof. The proof of the right-hand side inequality in (1) is direct. To prove the left-hand side one, we choose the boundary point $x_{0} \in \partial \Omega$ at which $\left|x-x_{0}\right|=\delta(x)$.

doi:10.5186/aasfm.2010.3542

2000 Mathematics Subject Classification: Primary 47B38, 42B35, 46E35.

Key words: Hardy inequality, weighted spaces, variable exponent. 
Then $|x-y| \leqq\left|x-x_{0}\right|+\left|x_{0}-y\right|$ which yields

$$
a_{\Omega}(x) \geqq \int_{\mathbf{R}^{n} \backslash \Omega} \frac{d y}{\left[\left|x_{0}-y\right|+\delta(x)\right]^{n+\alpha}} \geqq \int_{B\left(x_{0}, \delta(x)\right) \cap\left(\mathbf{R}^{n} \backslash \Omega\right)} \frac{d y}{\left[\left|x_{0}-y\right|+\delta(x)\right]^{n+\alpha}} .
$$

Since $\left|y-x_{0}\right| \leqq \delta(x)$, we have

$$
\int_{B\left(x_{0}, \delta(x)\right) \cap\left(\mathbf{R}^{n} \backslash \Omega\right)} \frac{d y}{\left[\left|x_{0}-y\right|+\delta(x)\right]^{n+\alpha}} \geqq \int_{B\left(x_{0}, \delta(x)\right) \cap\left(\mathbf{R}^{n} \backslash \Omega\right)} \frac{d y}{[2 \delta(x)]^{n+\alpha}} \geqq c_{2}[\delta(x)]^{-\alpha}
$$

where the last inequality comes from the assumption that $\Omega$ is of type $A^{c}$.

\section{Main result}

For the definition of $\mathscr{P}(\Omega), w$-Lip $(\Omega),(\alpha, p(\cdot))$-property and Strichartz condition, we refer to [1].

Theorem 3. Let $\Omega$ be a bounded open set in $\mathbf{R}^{n}, p \in \mathscr{P}(\Omega) \cap w$-Lip $(\Omega)$ and $0<\alpha<\min \left(1, \frac{n}{p_{+}}\right)$. Let $\Omega$ be of type $A^{c}$. Then the validity of the Hardy-type inequality

$$
\left\|\frac{1}{\delta(x)^{\alpha}} \int_{\Omega} \frac{\varphi(y)}{|x-y|^{n-\alpha}} d y\right\|_{p(\cdot)} \leqq C\|\varphi\|_{p(\cdot)}
$$

is equivalent to the $(\alpha, p(\cdot))$-property.

Proof. The result follows from Theorem 14 in [1] in view of Lemma 2 proved above.

Note that the following corollary is a new statement, in spite of the fact that it concerns the case of constant $p$; the known such statements for constant $p$ used other assumptions on the open set $\Omega$.

Corollary 4. The Hardy inequality

$$
\left\|\frac{1}{\delta(x)^{\alpha}} \int_{\Omega} \frac{\varphi(y)}{|x-y|^{n-\alpha}} d y\right\|_{p} \leqq C\|\varphi\|_{p}, \quad 1<p<1 / \alpha
$$

holds for any bounded open set $\Omega \subset \mathbf{R}^{n}$ satisfying the Strichartz condition and $A^{c}$ condition.

Proof. By Lemma 11 and Lemma 21 in [1], the set $\Omega$ has the $(\alpha, p(\cdot))$-property and then the statement follows from Theorem 3 .

Finally, we note that in the paper [1], in some statements the set $\Omega$ was assumed to be a domain, however the proofs never used the fact that it is connected.

\section{References}

[1] Rafeiro, H., and S. Samko: Hardy type inequality in variable Lebesgue spaces. - Ann. Acad. Sci. Fenn. Math. 34:1, 2009, 279-289. 